\section{Human genetics Human genetics discovering ourselves}

\section{Comai}

Heredity (2007) 99, 481-482; doi:10.1038/sj.hdy.6801060; published online 19 September 2007

G il: What are you, a man or a mouse? Dr H Hackenbush: You put a piece of cheese down there and you'll find out. (A Day at the Races, 1937)

Just as Gil wonders whether Dr H Hackenbush (Groucho Marx) is a primate or a rodent, we are concerned with differentiating humans from the other mammals. Like Groucho, scientists have strived to provide an objective test, but the task is proving challenging. It is not surprising, therefore, that when a paper reports the discovery of a gene potentially associated with human evolution it gets considerable attention: could it contribute to human identity? Such is the case for a recent report in Plos Genetics (Parker-Katiraee et al., 2007). The authors found a gene, Krueppel-like factor 14 (KLF14), encoding a putative regulator of the $\mathrm{Zn}$ finger-type that is maternally imprinted in mouse. Genetic differences between human populations suggest that it has been subjected to accelerated evolution or relaxed selection (Figure 1).

KLF14 resides on a region of chromosome 7 and is thought to affect autism and the dismorphic disease RussellSilver syndrome. KLF14 mRNA is expressed in many tissues, but more abundantly in embryos than in adults, suggesting a role in early development. By measuring the relative contribution of each parental allele, the authors determined that KLF14 is preferentially expressed from the maternally inherited copy. In mouse, maternal expression of Klf14 required the action of DNA methyltransferase Dnmt3a, but, surprisingly, no specialized chromatin modification such as DNA or histone methylation could be found at the Klf14 locus, as expected for a gene that is epigenetically controlled by imprinting. The authors speculated that a linked, but distant imprinting centre may be responsible, possibly the one known to imprint the close MEST gene. Interestingly, KLF14 is intronless, suggesting that it may have originated from accidental reverse transcription of the mRNA of a related gene. Consistent with a duplication close to the time of mammalian radiation, KLF14 is absent in marsupials but present in the same chromosomal context in all sampled eutherians.

Is this gene important for human evolution? That is not easy to ascertain. Comparing our genes to those of a chimpanzee, it is difficult to understand why we write poems and surf the internet while they find different occupations. This is because our genes are essentially identical. Or are they? Many small differences exist between the two genomes. Presumably, some of these changes bring sufficient variation to the developmental program of the two organisms to account for the different outcome. Scientists have attempted to identify the DNA changes, or polymorphisms, that really matter: most of the changes underlying the evolution of humans from a primate common ancestor are expected to affect expression or function of gene products. In many cases, positive selection-a directional evolutionary force favouring novel useful alleles-could have contributed to their establishment in the population. However, the distinction between changes that are functional (established by selection) and neutral (established by random events such as genetic drift) is often unclear.

Statistical tests attempt to determine whether variation in a gene defies a null hypothesis of neutrality. For example,

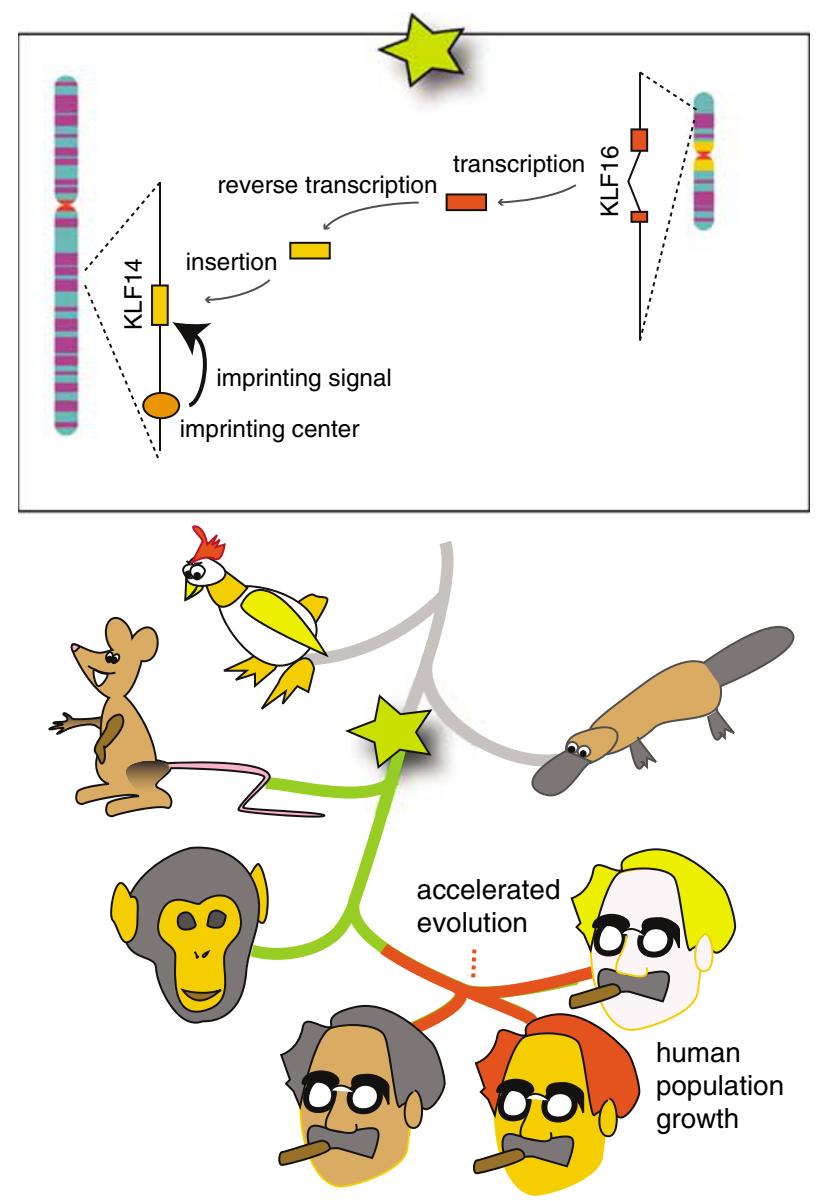

Figure 1 Inferred history of the KLF14 gene. The box at the top illustrates a hypothetical duplication of the ancestral KLF16 gene via reverse transcription and insertion near a preexisting imprinting center. The timing of this event is marked on the phylogenetic tree (star). The time of accelerated evolution is marked in red. 
non-synonymous DNA changes that result in protein sequence changes should be more common in a positively selected gene than silent changes (synonymous). However, the occurrence and preponderance of such tell-tale changes in alleles of a human gene must be evaluated for significance, for example, by comparison with a sample from a related taxon. But, the devil is the detail: tests for positive selection are clouded by the age of the selective event, by the demography of the studied populations (for example, there are more humans than chimps) and by the difficulty in ascertaining the rate at which mutations and recombination pepper and scramble different genes (Wright and Gaut, 2005). The human KLF14 gene appears to be more variable than the counterpart in primates, it had diverged between different human populations, and it has an unusually high number of non-synonymous changes, suggesting the possibility of positive selection. The authors looked for statistically significant evidence for positive selection, but found none. They could demonstrate, nevertheless, that the human gene is evolving faster than its counterpart in primates.

What is the likelihood that this imprinted gene, which has evolved rapidly in humans, but not in monkeys, is significant for human evolution? The imprinting behavior is tantalizing, yet it is not unique to humans. Indeed, while imprinting is often explained as the outcome of conflicting parental interests (mother loves all her children, father only those he sired), it can also be explained in different ways. One explanation is that imprinting reduces the imbalance produced by selective duplication of a stoichiometrically constrained (Veitia, 2004) regulatory factor (Walter and Paulsen, 2003). As noted by the authors, KLF14 does indeed appear to have originated by a duplication event and to be a regulatory factor. But, why has there been such rapid evolution in humans? Could this gene have contributed to the witty intelligence of Groucho? Possibly, but unlikely, says the sceptic lobe of our brain. Instead, KLF14 function could have responded to more mundane selection acting on early humans, be it campfire smoke, new diets or pathogens. Of particular interest is the possibility that KLF14 may contribute to physiological disease susceptibility in humans. The authors found no association of KLF14 alleles to autism or Russell-Silver syndrome, although it is possible that susceptibility to disease derives from polymorphism at a distal imprinting center. Such a hypothesis cannot be tested easily because, for obvious reasons, it is difficult to measure parental expression in affected tissues of patients. Instead, the genotype at candidate regions must be compared in healthy and diseased cohorts.

Not all rapidly evolving genes are the result of positive selection. Genes can evolve rapidly for many reasons, not least because they may not affect fitness at all, or are buffered from purifying selection by redundancy, or by being silenced (Barker et al., 2005). Yet, the KLF14 story is not a tale of 'Much ado about nothing'. A rapidly evolving imprinted locus is undeniably interesting and this study presents an admirable characterization. While the mechanisms underlying this pattern are mysterious, their elucidation will be worth waiting for. In the meantime, the search for effective methods to ferret out positive selection in the haystack of genomic information is on. Who are we? What kind of cheese should we put down there to find out?

L Comai is at the Department of Plant Biology and Genome Center, University of California at Davis, Davis, CA, USA.

e-mail: lcomai@ucdavis.edu

Barker MS, Demuth JP, Wade MJ (2005). Maternal expression relaxes constraint on innovation of the anterior determinant, bicoid. PLoS Genet 1: e57.

Parker-Katiraee L, Carson AR, Yamada T, Arnaud P, Feil R, Abu-Amero SN et al. (2007). Identification of the imprinted KLF14 transcription factor undergoing human-specific accelerated evolution. PLoS Genet 3: e65.

Veitia RA (2004). Gene dosage balance in cellular pathways: implications for dominance and gene duplicability. Genetics 168: 569-574.

Walter J, Paulsen M (2003). The potential role of gene duplications in the evolution of imprinting mechanisms. Hum Mol Genet 12: R215-R220.

Wright SI, Gaut BS (2005). Molecular population genetics and the search for adaptive evolution in plants. Mol Biol Evol 22: 506-519.

\section{Editor's suggested reading}

Balaresque P, Manni F, Dugoujon JM, CrousauRoy B, Heyer E (2006). Estimating sex-specific processes in human populations: are XYhomologous markers an effective tool? Heredity 96: 214-221.

de Knijff P (2006). The longevity of Y chromosomes: the human $Y$ chromosome is not dead (yet). Heredity 97: 377-378

Oldham MC, Geschwind DH (2006). Comparative genomics: grasping human transcriptome evolution: what does it all mean? Heredity 96: 339-340.

Roy SW, Ferreira MU, Hartl DL (2006). Evolution of allelic dimorphism in malarial surface antigens. Heredity (advanced online publication, doi:10.1038/sj.hdy.6800887).

Torgerson DG, Singh RS (2006). Enhanced adaptive evolution of sperm-expressed genes on the mammalian X chromosome. Heredity 96: 39-44. 